\title{
L'imaginaire politique de la théorie de l'énonciation
}

\author{
François Provenzano \\ Université de Liège \\ adresse : Francois.Provenzano@ulg.ac.be
}

\section{Introduction}

Le présent article a pour objectif d'éclairer la manière dont une réflexion relative au langage peut être imprégnée d'un imaginaire politique. Il s'inscrit sur le terrain de l'histoire et de l'épistémologie de la linguistique par le biais de l'hypothèse suivante: le discours de la linguistique, comme d'ailleurs celui des autres disciplines scientifiques, est traversé par un imaginaire qui se lit dans les mots des linguistes. Ces mots sont envisagés ainsi dans la puissance de leur signifiant, et non uniquement comme des concepts bâtissant une théorie ${ }^{1}$. Nous reviendrons plus loin sur cette hypothèse et sur son application au cas de la linguistique de l'énonciation; il nous faut d'abord contextualiser rapidement l'objet de cette étude.

Dans l'histoire des idées linguistiques, le tournant des années 19601970 est marqué en France par le paradigme structuraliste saussurien, indexé, notamment par l'avant-garde Tel Quel (Jenny, 2008 : 181 s.), sur la théorie marxiste pour servir une critique de l'ordre social bourgeois. Cette articulation du structuralisme et du marxisme, parfois problématique et

1. Ce terrain est, en ce qui nous concerne, relativement neuf. Nous l'abordons depuis peu avec deux autres collègues de l'Université de Liège, Sémir Badir et Stéphane Polis, avec qui nous formons le collectif «Lttr13" (voir bibliographie en fin d'article). Certaines idées de cette contribution ont donc une genèse collective; cela dit, il va de soi que nous assumons personnellement l'hypothèse qui sera développée ici. 
mouvementée, est une question déjà bien explorée, tant du point de vue strictement théorique (depuis les contemporains comme Louis Althusser et Lucien Sebag, jusqu'aux travaux de Thomas Pavel) que du point de vue socio-historique adopté par exemple par François Dosse ou Frédérique Matonti dans plusieurs travaux récents (voir bibliographie en fin d'article). Nous ne nous attarderons pas ici sur ces questions, qui nous servent plutôt de contrepoint pour porter notre attention sur l'émergence, dans les mêmes années, d'un autre paradigme, celui de la linguistique de l'énonciation, et interroger son articulation au politique.

Attaché principalement aux deux figures fondatrices d'Émile Benveniste et d'Antoine Culioli, ce paradigme se trouve actuellement au centre de nombreux travaux qui, non seulement s'en revendiquent, mais aussi en interrogent les fondements et les filiations. Le présent travail s'inscrit dans cette démarche génétique, mais tentera d'apporter sur ce moment de l'histoire des idées linguistiques un éclairage particulier, en postulant un lien imaginaire entre épistémologie et politique, plus particulièrement entre le projet de connaissance qui caractérise la linguistique énonciative (la manière dont il se formule) et le nouveau rapport au pouvoir tel qu'il traverse le discours social de la France de mai 1968.

\section{Le terme et son imaginaire}

Qu'entend-on exactement par lien imaginaire? Et qu'est-ce que la prise en compte de l'imaginaire peut apporter à l'histoire des idées linguistiques? Au sein du collectif LTTRI3, nous avons entrepris une enquête rhétorique sur la genèse et l'expansion de quelques termes phares de la discipline linguistique récente (énonciation, subjectivité, intersubjectivité) et proposons d'englober sous l'étiquette d'imaginaire d'un terme, sous bénéfice d'un inventaire plus raisonné, tout ce qui, parallèlement à son usage théorique, a trait aux valeurs connotatives ou rhétoriques (argumentatives, esthétiques, éthiques, sociologiques) qui peuvent aider à son émergence et à sa reconnaissance, et qui contribuent aussi, finalement, à son déclin et à son rejet. Ce qui nous intéresse ici c'est donc la puissance d'un Signifiant: puissance interne que peut déceler une recherche étymologique (en ce compris l'étymologie populaire), génétique (sur la " lignée " terminologique à laquelle il appartient), morpho-syntaxique (sur la dérivation, les constructions et collocations lexicales qu'il permet), etc. Puissance également externe selon les cercles de notoriété et de diffusion auxquels il accède. Le Signifiant n'est pas roi pour autant. Il est évident que le terme demeure tributaire d'une conceptualisation et que son signifié conserve une prégnance première. Cependant, au lieu 
de penser selon une généalogie des concepts, nous proposons de penser une génétique rhétorique des termes qui, même si bien sûr elle ne dira pas le fin mot sur la fortune d'un terme, n'en constitue pas moins une approche possible, selon nous trop peu considérée.

L'emploi de ce concept d'imaginaire fait inévitablement penser aux travaux menés depuis la fin des années 1970 par Anne-Marie Houdebine et auxquels est attaché le concept d'Imaginaire linguistique ${ }^{2}$. Centré dans un premier temps sur le rapport entretenu par les sujets parlant avec leur langue, ce concept s'est élargi aux constructions théoriques elles-mêmes, ce qui fait dire à Houdebine: " la version qu'on donnera de la langue n'est que faite d'imaginaire. Cela même dans les travaux supposés scientifiques des linguistes. " (Houdebine-Gravaud, 2002 : 14) ${ }^{3}$ Nous souscrivons entièrement à ce postulat capital, en lui donnant cependant une orientation particulière. Tandis que le concept d'Houdebine invite à considérer l'interaction d'une série de normes, qui trouvent elles-mêmes leur genèse dans un imaginaire social particulier, nous centrons notre attention sur des termes et, plus particulièrement, sur ce qui, dans ces termes, relie la conceptualisation linguistique à un imaginaire politique. Celui-ci ne se traduit pas nécessairement en normes d'appréciation linguistique; nous dirons plutôt que c'est la théorie linguistique qui peut à nos yeux être reconduite à des principes de vision de la société et des rapports entre l'individu et la société.

Plusieurs raisons nous font croire qu'une telle approche est particulièrement indiquée pour le cas du terme énonciation. Sans pouvoir en faire l'exposé ici, rappelons simplement que ce terme ne se laisse pas ramener aujourd'hui à un simple désignant. Dès Benveniste, il essaime dans le travail du linguiste comme s'il rencontrait à chaque fois des conceptualisations en cours, pas forcément abouties, non pour baliser fermement ces conceptualisations, mais plutôt pour favoriser leur ouverture à des significations autres que conceptuelles. C'est ce travail terminologique particulier que nous appelons les fonctions imaginaires du terme.

Parmi celles-ci, c'est donc la fonction politique qui va nous retenir ici, c'est-à-dire la rencontre entre le terme et, par le biais de ses constellations terminologiques, un air du temps qui lui permet de trouver une incidence inattendue, qui le fait, au sein de la théorie, véhicule de significations socio-politiques.

2. Dès Houdebine 1979 et jusqu’à Houdebine-Gravaud (dir.) 2002.

3. Cette veine d'investigation sur l'imaginaire linguistique des linguistes a notamment été exploitée dans les travaux de Ferenc Fodor sur Antoine Meillet (voir par exemple Fodor 1996). 
Une telle lecture n'a donc pour ambition ni de mettre au jour les sources textuelles de la théorie de l'énonciation pour y chercher un quelconque substrat politique, ni de refléter les engagements politiques effectifs des théoriciens envisagés. Que Benveniste et Culioli aient affiché quelque proximité avec la pensée marxiste a finalement peu d'importance ici; ni philologie ni sociologie, notre démarche prend pour objet la théorie comme rhétorique inscrite dans un discours social et partageant avec lui certaines portions d'imaginaire.

\section{La théorie de l'énonciation et la lecture politique}

Pour faire cependant encore un instant un peu de philologie et de sociologie, il faut dire que cette lecture politique de la linguistique de l'énonciation semble être à la fois découragée et encouragée par la proto-histoire de ce paradigme, étroitement associée dans les années 1920 et 1930 aux intellectuels soviétiques autour de Mikhail Bakhtine et, en particulier, à Valentin Vološinov, à qui l'on attribue aujourd'hui le fameux Marxisme et Philosophie du langage (Vološinov [1929] 2010).

Découragée d'abord parce que, à suivre Patrick Sériot (2011), la lecture marxiste de l'ouvrage alors attribué à Bakhtine est le produit d'une illusion d'optique, propre aux intellectuels français de la fin des années 1970, qui ne correspond nullement à l'orientation donnée par Vološinov à son travail ${ }^{4}$. En outre, cette orientation, que Sériot définit comme "psycho-socio-sémiotique ", ne rejoindrait nullement les présupposés théoriques de la démarche énonciativiste benvenistienne, en ceci - principalement - qu'on ne trouve aucune « théorie du sujet » chez Vološinov (Sériot, $2011: 92$ ).

On ne peut qu'être convaincu par la fine lecture théorique de Sériot. Remarquons cependant que, dans le même geste par lequel il réfute les lectures marxiste et benvenistienne de Vološinov, l'auteur nous montre pourtant ce qui participe chez lui d'un imaginaire politique. Par-delà leurs différences théoriques, cet imaginaire politique peut rassembler Vološinov et Benveniste dans une même conception du rapport entre le langage et la société. Gardons en mémoire cette idée selon laquelle, pour Vološinov, "l'individu "abstrait" n'a aucune existence en dehors du ou d'un "groupe social” " (Sériot, 2011 : 91), dont on trouve certains types, dit Vološinov,

4. «Les réflexions de V. Vološinov sur le langage, loin d'être l'anticipation originale d'une linguistique "marxiste", s'insèrent parfaitement dans une problématique propre à son époque, à savoir la réaction anti-positiviste qui se manifestait, depuis la fin du XIX siècle, en Allemagne et en Russie beaucoup plus nettement qu'en France. » (Sériot $2011: 86$ ) 
" dans les veillées de village, les fêtes populaires en ville, le bavardage des ouvriers pendant la pause à l'heure du déjeuner, etc. » (Vološinov, [1929] 2010 : 99; cité dans Sériot, 2011 : 91). L'échange verbal comme fondement de l'existence sociale de l'individu" a pour corollaire, "lorsqu'une personne a perdu son auditeur intérieur ", "la chute idéologique de la personne hors de son milieu de classe " (Vološinov, 1930 : 71; cité dans Sériot, 2011 : 92).

Le propos de Vološinov est bien de situer la réalité fondamentale de l'activité de langage - et du même coup l'existence sociale de l'individu dans l'interaction sociale concrète, ce que Benveniste fera dans des termes presque identiques quarante ans plus tard:

La réalité effective du langage n'est pas un système abstrait de formes linguistiques, ni un énoncé monologique isolé, ni l'acte psycho-physiologique de réalisation de l'énoncé, mais l'événement social de l'interaction verbale, réalisé dans l'énoncé et les énoncés. C'est l'interaction verbale qui constitue, ainsi, la réalité fondamentale du langage. (Vološinov, [1929] 2010 : 319; nous soulignons)

Ce rapport fondateur chez Vološinov entre l'activité de langage et l'inscription de l'individu dans une certaine structure sociale est bien mis en évidence par Jean-Paul Bronckart et Cristian Bota qui, dans leur Bakhtine démasqué, soulignent quant à eux l'" ancrage résolument marxiste » de Vološinov (Bronckart \& Bota, 2011 : 415). Ce n’est pas tant cet étiquetage marxiste qui nous intéresse, que l'idée selon laquelle l'individu humain n'accède à l'existence qu'à travers « la place qu'il occupe dans l'organisation d'un groupe, c'est-à-dire par sa position dans la configuration des classes sociales " (ibid.) et l'affirmation que " les dimensions langagières et sémiotiques jou[ent] un rôle décisif dans la construction même des entités sociales et idéelles " (ibid. : 416). Même si ces conceptions sont loin d'être conformes au marxisme le plus orthodoxe, on ne niera pas qu'elles participent tout de même d'un imaginaire politique.

Ce même imaginaire politique, Gérard Dessons et Jean-Claude Milner s'emploient à le mettre en évidence - quoique de manière un peu allusive - dans les travaux d'Émile Benveniste. Dans son Émile Benveniste, linvention du discours, Dessons note que

[ ] les fonctions de repérage propres à l'énonciation, par lesquelles chaque parlant " déploie un réseau complexe de relations spatio-temporelles qui déterminent les modes d'énonciation " [Benveniste, 1974 : 99], pourront

5. Voir encore cette citation: «L'essence véritable du langage est l'événement social de l'interaction verbale, réalisé par l'énoncé » (Vološinov, 1930: 66; cité dans Sériot 2011: 92). 
être mises en relation avec la production des valeurs réglant la vie sociale des individus. On touche à la dimension proprement politique du langage, donnée en même temps que sa dimension éthique, la société se trouvant mise nécessairement en relation avec l'individu lors de chaque instance de discours. (Dessons, $2006: 111$ )

D’une manière comparable, Jean-Claude Milner met en lumière le fondement hégélien de la réflexion de Benveniste, dont la théorie de l'énonciation ferait du langage "l'infrastructure matérielle de la subjectivité " (Milner, 2008 : 131).

Ce sont sur ces pistes que nous nous engageons à présent, en tentant de retracer le fil de cet imaginaire politique, d'abord à la marge des articles canoniques de Benveniste sur l'énonciation, c'est-à-dire dans le Vocabulaire des institutions indo-européennes (Benveniste 1969), ensuite dans les deux entretiens qu'il donne à des revues non spécialisées ${ }^{6}$.

\section{Benveniste politique}

Dans son examen du vocabulaire indo-européen consacré au droit (ibid. : t. II, livre 2), Benveniste met clairement en lumière le lien qu'il constate entre le pouvoir et la parole:

Nous avons constaté une relation fréquente entre des termes servant à qualifier des institutions et des verbes marquant d'une manière ou d'une autre la notion de «dire". De l'acte de parole au droit, à la règle, tels qu'ils organisent certaines fonctions sociales, il y a souvent un rapport étroit. En particulier, des institutions politiques sont parfois dénommées par une spécification de la notion de " parole " dans le sens d' " autorité " (ibid. : 143).

Cette constatation est nourrie par deux enquêtes terminologiques portant sur deux domaines distincts, mais ayant en commun un certain usage de la parole proférée: d'une part le droit divin mais aussi, on va le voir, le fondement même de la personnalité humaine; d'autre part l'autorité politique en tant que police, c'est-à-dire en tant qu'elle assigne une place sociale bien définie, plus particulièrement sur la base d'une échelle économique.

6. Plusieurs aspects de notre démarche sont également redevables au travail de Aya Ono (2007) - qui cependant s'intéresse plus résolument aux bougés conceptuels, et non aux maillages terminologiques, autour de la notion d'énonciation -, ainsi qu'au travail de Chloé Laplantine (2011), qui s’intéresse à la poétique ébauchée par Benveniste à propos de Baudelaire et dont les conclusions font écho à la « libération du sujet » qui se dessine en filigrane des motifs que nous allons tenter de mettre en évidence dans les pages qui suivent. 
Pour expliquer le rapport entre la notion de « parler » et celle de « droit divin ", Benveniste fait intervenir la forme latine infans, "l'enfant en bas âge, celui qui ne parle pas ", pour montrer qu' " on vise ainsi la parole articulée, l'acte de parole comme manifestation du langage, comme émanation de la personne humaine " (ibid.: 137), mais aussi comme "manifestation d'une faculté impersonnelle, commune à tous les êtres humains, le fait qu'ils soient capables de parole" (ibid.: 138). Dès lors, "vox populi, vox dei, la "voix" du peuple a quelque chose de divin. C'est pourquoi aussi le fatum est une énonciation qui n'a pas de source personnelle, qui n'est pas rapportée à un homme et qui tire de cette origine supra-humaine quelque chose de mystérieux, de fatal, de décisif. " (ibid.: 139). L'examen de cette racine ${ }^{*} b h \bar{a}$-dans le vocabulaire indo-européen conduit ainsi Benveniste à considérer d'un même regard l'activité de parole de l'être humain, sa manifestation collective dépersonnalisée et le " pouvoir étrange " qui y est associé.

L'autre piste terminologique rejoint et complète le premier fragment d'imaginaire politique; elle concerne la parole comme " dire autorisé ", comme police sociale, et prend pour point de départ le lien problématique du latin censor avec ses dérivés: "Le censor est un magistrat, mais le verbe censeo ne signifie rien de plus que "estimer, juger, énoncer un avis"; tandis que le census est une opération technique: estimation de la fortune et classement des citoyens. " (ibid.: 143-144). Après l'examen d'autres attestations de la même racine dans d'autres langues indo-européennes, l'articulation du domaine sémantique se précise: "Celui qui "parle" ainsi est en position souveraine; en déclarant ce qui est, il le fixe; il énonce solennellement ce qui s'impose, la vérité du fait ou du devoir. " (ibid. : 147). Benveniste met ainsi en évidence le caractère fondamentalement performatif de cette parole autorisée: "Toute parole prononcée avec l'autorité détermine un changement dans le monde, crée quelque chose []. Des valeurs obscures et puissantes demeurent dans cette auctoritas, ce don réservé à peu d'hommes de faire surgir quelque chose et - à la lettre - de produire à l'existence. » (ibid.: 150-151)

Ici encore, il faut garder en mémoire ces formulations - dont on devine à la lecture qu' elles portent la trace d'une certaine fascination chez celui qui les a produites -, car elles concentrent les termes où précipite l'imaginaire politique de la théorie de l'énonciation chez Benveniste. User de la parole peut vouloir dire "faire exister dans la société »; faire exister un sujet certes, mais ce pouvoir proprement magique ne trouve tout son sens que lorsqu'il est rapporté à la collectivité humaine au plus 
haut degré de sa généralité. En s'emparant de l'autorité du censor, cette collectivité change le monde dans lequel elle vit.

Changer le monde, réinventer la société, c'est bien l'un des mots d'ordre de mai 1968, période qui coïncide précisément avec deux longs entretiens que Benveniste donne à deux périodiques situés hors du champ strictement académique et résolument à gauche sur l'échiquier idéologique: Les Lettres françaises, soutenues par le Parti Communiste, alors dirigées par Aragon, et Le Nouvel Observateur .

On y trouve de manière générale un matériau propice au type d'investigation que nous défendons, à savoir une observation des termes comme symptômes d'un imaginaire. Contrairement aux publications spécialisées, les textes plus informels laissent volontiers affleurer des occurrences ou des rapprochements terminologiques qui auraient pu résister à la formulation dans un contexte de publication plus contrôlé.

Pour faire écho aux remarques par lesquelles nous avons ouvert notre propos, on remarquera d'emblée que Benveniste situe sa conception de la linguistique à rebours de ce que défend alors une certaine doxa structuraliste à la mode: si la linguistique peut être une science pilote pour une culturologie, "ce n'est pas du tout en vertu d'une supériorité intrinsèque, mais simplement parce que nous sommes avec la langue au fondement de toute vie de relation" (Benveniste, 1974 : 26).

Ce rôle du langage comme fondement de l'interaction sociale - voilà déjà un premier écho à Vološinov - est complété ici par la dimension existentielle du langage pour l'homme, qui fait à chaque fois l'expérience singulière de sa réinvention: " [ ] tout homme invente sa langue et l'invente toute sa vie. Et tous les hommes inventent leur propre langue sur l'instant et chacun d'une façon distinctive, et chaque fois d'une façon nouvelle. Dire bonjour tous les jours de sa vie à quelqu'un, c'est chaque fois une réinvention. » (Benveniste, 1974 : 18-19) On notera l'insistance placée sur l'inventivité attribuée aux actes verbaux les plus anodins et les plus concrets, mais aussi la généralisation de cette propriété à " tous les hommes".

Après invention, nous touchons ici du doigt l'un des termes clés de l'imaginaire politique de la réflexion de Benveniste, celui d'appropriation. Nous connaissons le passage célèbre de l'article « L'appareil formel de

7. "Structuralisme et linguistique ", entretien de Pierre Daix avec Émile Benveniste. Les Lettres françaises, 1242, 24 au 24 juillet 1968, pp. 10-13; repris dans Benveniste 1974 : 11-28 (c'est à cette édition que nous nous référerons); "Ce langage qui fait l'histoire ", propos recueillis par Guy Dumur dans Le Nouvel Observateur, spécial littéraire, $\mathrm{n}^{\circ} 210$ bis (20 novembre au 20 décembre 1968), pp. 28-24; repris dans Benveniste 1974 : 29-40 (c'est à cette édition que nous nous référerons). 
l'énonciation ", où l'auteur définit cette notion " comme un procès d'appropriation" (Benveniste, 1974 : 82). L'intérêt est de voir que ce terme est précisément celui dont le signifiant insiste dans l'entretien donné aux Lettres françaises, où le linguiste souligne avec emphase l'importance de l'enjeu:

L'appropriation du langage à l'homme, c'est l'appropriation du langage à l'ensemble des données qu'il est censé traduire, l'appropriation de la langue à toutes les conquêtes intellectuelles que le maniement de la langue permet. C'est là quelque chose de fondamental: le processus dynamique de la langue, qui permet d'inventer de nouveaux concepts et par conséquent de refaire la langue, sur elle-même en quelque sorte. Eh bien! tout cela c'est le domaine du «sens ». (Benveniste, 1974: 21)

L'insistance de ce terme appelle, entre parenthèses, une rapide mise au point préalable sur le caractère individualiste attribué à la théorie de l'énonciation, souvent perçue comme une théorie du sujet d'énonciation, de l'individu qui, en s'appropriant la langue, devient sujet singulier. Cette interprétation est évidemment problématique pour indexer la pensée de Benveniste sur un imaginaire politique de gauche. C'est d'ailleurs la critique que lui adresse l'analyse du discours (de Michel Pêcheux), dont le propos est précisément de désubjectiver l'énonciation et de mettre au centre du fonctionnement du discours la notion d'intertexte ${ }^{8}$. C'est oublier que, pour Benveniste, cette manière de présenter les choses n'est pas un renforcement de la singularité du sujet, mais bien une tentative de dépassement de l'opposition entre l'individu et la société (voir Milner, 2008 : 128-129; Dessons, 2006 : 107) : "En vertu de la polarité je: tu, individu et société ne sont plus des termes contradictoires, mais termes complémentaires. " (Benveniste, 1966 : 25) Comme le dit encore Pascal Michon, «[d] u point de vue de la langue, il n'existe donc pas de dualisme entre subjectivation et sociation" (Michon, 2010 : 113). Quoi qu'il en soit de la lecture que l'analyse du discours fera de Benveniste, elle prouve en tout cas que ce discours était perçu comme investi d'une charge politique (même si, en l'occurrence, il s'agissait de marxiser davantage le propos). La complexité tient précisément à ce que la théorie linguistique de Benveniste ne peut pas vraiment être indexée sur la pensée marxiste, mais se présente plutôt comme les résultats d'une importante médiation à partir de ce substrat politique.

Loin de s'indexer sur une position strictement individualiste - ce que peut laisser penser la définition de "L'appareil formel de l'énonciation » qui insiste sur « le locuteur »-, le propos de Benveniste donne donc à ce

8. Dominique Maingueneau, Intervention au colloque «Les théories de l'énonciation: Benveniste après un demi-siècle ", Université Paris-Est Marne-la-Vallée, 23 au 23 novembre 2011. 
terme appropriation une résonance résolument collective: c'est l'Homme dans sa généralité qui est invité à s’approprier une langue qui lui donnera la capacité créatrice de s'inventer dans la société ${ }^{9}$. La propriété des censores romains est rendue ici à la multitude sociale sans distinction, qui récupère les moyens de sa production sémantique ${ }^{10}$.

Cette appropriation-réinvention est donc aussi, et inséparablement, une action sur le monde. En écho à son interprétation de l'infans dans le Vocabulaire des institutions indo-européennes, Benveniste reprend ici l'exemple de l'enfant qui acquiert le langage pour expliquer qu' " [e]n apprenant le nom d'une chose, il acquiert le moyen d'obtenir cette chose. En employant le mot, il agit donc sur le monde et s'en rend compte obscurément très tôt " (Benveniste, 1974 : 24). L'appropriation langagière est ici explicitement associée à la propriété des biens matériels et Benveniste la formule comme la prise de conscience d'un pouvoir qui, à la fois, est mystérieux et pourtant constitue l'évidence du rapport de l'homme au monde.

Au passage, on notera encore que c'est précisément ce terme appropriation que soulignera Roland Barthes dans son commentaire enthousiaste de Benveniste:

L'énonciation n'est pas l'énoncé, bien sûr; et elle n'est pas non plus (proposition plus subtile et plus révolutionnaire) la simple présence de la subjectivité dans le discours; elle est l'acte, renouvelé, par lequel le locuteur prend possession de la langue (se l'approprie, dit justement Benveniste) [...]. (Barthes, 1974 : 194-195)

La qualification plus ou moins " révolutionnaire " ${ }^{11}$, jointe à l'action de «s'approprier » qui distingue l'énonciation, ne peut manquer de renvoyer

9. Et d'inventer du même coup la société elle-même. La récente édition des Dernières leçons de Benveniste au Collège de France témoigne de cette préoccupation du linguiste de concevoir l'existence comme une existence forcément sociale: " Nous sommes parti de cette constatation que la langue entière est informée et articulée par la signification. Elle ne pourrait fonctionner autrement et c'est d'ailleurs sa raison d'être; sans quoi il n'y aurait, à un bout, pas de pensée, à l'autre bout, pas de société, donc pas d'être, et personne pour le constater." (Benveniste 2012 : 141)

10. Cette résonance collective du terme appropriation est à lire également dans l'article "Structure de la langue et structure de la société » (Benveniste 1974 : 100): «chaque classe sociale s'approprie des termes généraux, leur attribue des références spécifiques et les adapte ainsi à sa propre sphère d'intérêt [ ]. "

11. Ce terme nous offre au passage l'occasion d'un saut intertextuel avec le fameux manifeste surréaliste La Révolution d'abord et toujours (1925), signé par Benveniste; dans sa préface aux Dernières leçons, Julia Kristeva en rappelle ce passage, qui fait écho avec nos développements: «"Nous [...] ne la [la Révolution] concevons que sous sa forme sociale [...] l'idée de Révolution est la sauvegarde la meilleure et la plus efficace de l'individu. " (cité par Kristeva 2012 : 37) 
à un imaginaire de la lutte politique et de faire dès lors du terme l'un des mots de passe culturels qui polarisent alors le champ idéologique français. La mention de " dit justement " participe pleinement d'une sublimation $\mathrm{du}$ linguistique vers l'imaginaire politique et la modalisation du discours rapporté compte tout autant que ce discours lui-même et que sa source: Barthes impose l'expression comme, c'est-à-dire comme ajustée à ce que la notion d'énonciation peut donner à comprendre des rapports entre l'individu et les structures sociales.

Reste que ce terme d'appropriation reçoit chez Benveniste toute sa charge d'imaginaire politique lorsque l'auteur se laisse aller à ébaucher lui-même la manière dont sa théorisation du fonctionnement de la langue pourrait embrasser la conceptualisation marxiste des rapports sociaux:

Il y a, en particulier, une manière d'organiser des rapports logiques qui apparaît très tôt chez l'enfant. [ ] Ce réseau complexe se retrouverait à un niveau profond dans les grandes démarches intellectuelles, dans la structure des mathématiques, dans les relations qui sont au fondement de la société. Je pense que certains des concepts marxistes pourraient à leur tour entrer peu à peu, une fois dûment élaborés, dans ce cercle de notions articulées par les mêmes rapports de base dont la langue offre l'image la plus aisément analysable. Mais j’ai tort de parler de tout cela comme de théories déjà exposées qu'il n'y aurait qu'à chercher dans un livre, alors que ce sont des choses auxquelles je réfléchis, mais qui sont encore en cours d'élaboration. (Benveniste, 1974 : 25 ; nous soulignons)

L'auteur livre ici les linéaments d'une pensée en cours, un état préformulé de sa réflexion, qui laisse encore coaguler l'ancrage disciplinaire avec l'imaginaire politique. C'est la même perméabilité discursive qui caractérise le finale de l'entretien, où se tient un discours sur la société du temps, qui semble éclairer par un biais particulier la théorie linguistique défendue, notamment à travers la notion de "prise de conscience "; l'intérêt est ici encore de voir un Benveniste qui " cherche ses mots ", qui passe d'un sujet à l'autre, d'un discours sur l'art qui semble en fait servir de point de passage entre son discours métalinguistique et son discours politique:

D'une façon générale nous sommes à l'époque des prises de conscience. [ ] je crois qu'au fond c'est là que nous rencontrons le problème que la langue nous a appris à voir. De même que nous ne parlons pas au hasard, je veux dire sans cadre, que nous ne produisons pas la langue hors de certains cadres, de certains schémas que nous possédons, de même je crois que l'art ne se produit pas non plus en dehors de cadres ou de schémas différents mais qui existent aussi. Et qui se reformulent ou qui renaissent dans la mesure même où l'on prend conscience de ce qui est périmé. 
Cette prise de conscience c'est déjà une voie ouverte vers le nouveau siècle. [ ] je crois que l'époque favorise ces prises de conscience du fait même que tant de valeurs acceptées se trouvent remises en question, et jusqu'aux systèmes de production. (Benveniste, 1974 : 28; nous soulignons)

Nous sommes ici au point de jonction des harmoniques que nous avons tenté de mettre en évidence, essentiellement autour des termes appropriation et prise de conscience. Il faudrait évidemment compléter cette enquête par un examen d'autres travaux de Benveniste, notamment les articles canoniques sur l'énonciation, qui mettrait sans doute en lumière d'autres constellations terminologiques. On peut penser par exemple à la série des termes, essentiels dans la théorie de l'énonciation, qui s'établit en relation avec la valeur du mot institution - instituer, instaurer, instant, instance - et que Gérard Dessons (2006) invite à lire en référence au Vocabulaire des institutions indo-européennes comme signe de " la cohérence d'une pensée qui ne dissocie pas langage et subjectivité ", une " pensée du présent dans le langage comme fondement de l'historicité et de la subjectivité » (Dessons, 2006 : 53). On peut penser également au fonctionnement terminologique du mot appareil dans les discours de Benveniste, encore un mot qui, l'année même où Louis Althusser publie son célèbre article sur les " appareils idéologiques d'État " (1970), ne peut manquer de connoter un intertexte politique. On se reportera pour s'en convaincre à la belle collecte que Maurice Tournier a fait des Mots de mai 1968, au sein desquels, nous dit-il, le mot appareil ne constitue pas un "désignant neutre ", mais bien un " terme marqué », " [m]arqueur des blocages de la société " (Tournier, 2007 : 18-19).

Mais Benveniste lui-même fait circuler ce terme dans son propre travail. Parmi les brouillons que nous donnent à lire les récentes découvertes d'Irène Fenoglio (2011), on trouve cette note préparatoire - relative à un autre article ( Le langage et l'expérience humaine ») et qui disparaîtra dans la version finale - où Benveniste laisse affleurer le terme d'appareil dans un passage qui en active les propriétés de dissimulation : «On la [la catégorie du temps] croit directement saisissable et < éludant toute saisie directe $>$ elle se cache dans un appareil formel qui la dissimule plutôt qu'il ne la démontre. " (Benveniste Pap.Or. 46, Env. 139, f 515, cité d'après Fenoglio, 2011 : 283). Cette note me paraît éclairer, dans les sous-couches du travail du linguiste, le point de jonction entre le sens idéologique bien connu du mot appareil et l'usage qu'en fait Benveniste dans sa théorie linguistique.

Le travail d'Irène Fenoglio sur les brouillons de cet article séminal extrêmement dense met au jour encore d'autres aspects de la genèse de 
cette pensée qui intéressent notre propos, dans cette écriture de soi à soi, qui ne s'est pas encore dépouillée, par la systématisation théorique, de son lien avec l'imaginaire de l'auteur ${ }^{12}$.

On remarque, entre bien d'autres choses, l'insistance graphique du mot appropriation, omniprésent, directement associé à la notion d'énonciation et souligné abondamment par Benveniste, qui glose notamment les " pronoms personnels, index, temps du verbe " comme des « instruments de l'appropriation » (Fenoglio, 2011 : 278-279).

On trouve également sous la main du Benveniste des brouillons le vaste intertexte qui relie sa théorisation de l'énonciation aux considérations étymologiques du Vocabulaire, évoquées plus haut dans cet article. On voit en effet clairement que la pensée de l'énonciation s'y construit par le biais de la performativité socio-politique que cette notion peut revêtir:

L'énonciation nombre de ses termes est un a une fonction créatrice quand elle répond à une fonction officiel $[$ sic $]$ quand elle est acte d'autorité! / Cela consiste à dénommer par un < l' > acte de parole en énonciation personnelle $=$ je déclare je jure ; à dénommer la décision : je décide que; je décrète; à dénommer la dénomin nomination: je vous nomme < prof chevalier ambassadeur > [...]. (Benveniste Pap. Or. 51, Env. 198, fo 482 [s.d.] ; cité d'après Fenoglio, 2011 : 293)

Dans cet autre passage, on retrouve les termes presque exacts par lesquels Benveniste commentait, en s'en étonnant, la puissance du censor romain, ici attribuée au procès de l'énonciation dans toute sa généralité:

En réalité c'est un changement, non, pas un changement dans la matière même de la langue. Un changement < plus subtil, plus profond > du fait qu'elle est mise en mouvement, que quelqu'un s'en est emparé et qu'elle la meut, la met en action, que cet appareil qui gisait, potentiel, mais inerte, consistant en signes d'un côté (signes lexicaux et autres), en modèles flexionnels et syntaxiques de l'autre < s'anime soudain devient soudain actuel $>$ prend soudain existence $<$ se forme en discours restituant autour de lui un mouvement vivant > ; te langure. Quelque chose naît au monde alors. Un homme s'exprime (lat. exprimere "faire sortir en pressant, faire jaillir à l'extérieur"), il fait jaillir la langue dans l'énonciation. (Benveniste, Pap. Or 51, Env. 198, fo 486; cité dans Fenoglio, 2011 : 301).

12. Fenoglio définit d'ailleurs sa démarche à partir d'une interrogation qui rejoint finalement l'objectif de cette contribution: « la visibilité de l'élaboration conceptuelle par et à l'intérieur de la chair de l'écriture (les mots, les phrases, le discours mais aussi, pour le discours en train de se fabriquer, les ratures, les reprises, les déplacements) est-elle de nature à modifier la compréhension des notions et concepts offerts dans le discours théorique publié? » (Fenoglio 2011 : 268) 
Le « don réservé à peu d'hommes de faire surgir quelque chose et - à la lettre - de produire à l'existence " (Benveniste, 1969 : 150-151) est ici rendu à l'homme, moteur d'un changement radical, inattendu, inespéré, inaugural, une épiphanie qui résulte d'un acte d'appropriation ("quelqu'un s'en est emparé »), qui est tout autant une manière de secouer le poids d'un appareil «inerte».

Enfin, de l'article "L'appareil formel de l'énonciation » (dans sa version publiée cette fois), nous retiendrons encore l'écho évident qu'il présente avec les propos de Vološinov déjà cités. Répétons-le: il ne s’agit pas de contester ici la disparité des programmes théoriques, mais plutôt de repérer ce qui, dans les marges de ces conceptualisations, se nourrit d'un même imaginaire.

Pour illustrer les multiples variétés de la situation de dialogue, Benveniste cite le travail de l'anthropologue Malinowski, qui envisage sous le nom de communion phatique " un procès où le discours, sous la forme d'un dialogue, fonde un [r]apport entre les individus "; voici la citation que Benveniste rapporte de Malinowski:

Il vaut la peine de citer quelques passages de cette analyse: [ ] Quand les gens s'assoient ensemble auprès d'un feu de village après avoir achevé leur tâche quotidienne ou quand ils causent pour se délasser du travail, ou quand ils accompagnent un travail simplement manuel d'un bavardage sans rapport avec ce qu'ils font, il est clair qu'ici nous avons affaire à une autre manière d'employer la langue, avec un autre type de fonction du discours. [ ] la situation en tous ces cas est créée par l'échange de mots [ ]. (Benveniste, 1974: 87)

Par le biais de Malinowski, c'est la même référence à une culture populaire, voire ouvrière, qui est ici convoquée comme support de la réflexion - pour rappel, Vološinov parlait de "veillées de village », de " fêtes populaires en ville » et de " bavardage des ouvriers pendant la pause à l'heure du déjeuner ".

Bref, il nous semble qu'à lire Benveniste par le biais de ce maillage intertextuel, on ne peut nier que sa théorisation de l'énonciation est traversée par un imaginaire politique qui, s'il ne s'identifie pas avec le marxisme, n'en opère pas moins à partir de certains de ses termes ou de ses motifs, en une série de déplacements et de médiations qui donnent sa chair terminologique à la théorie linguistique.

On aura remarqué notre prudence: notre propos ne nous conduit pas à conclure sur l'adhésion de fond de Benveniste à la pensée marxiste (ou marxisante). Mis à part les quelques indices signalés plus haut, l'auteur lui-même ne donne d'ailleurs guère d'éléments explicites pour prouver 
ou infirmer une telle hypothèse, ce qui n'était pas l'objectif de ce travail. Nous avons plutôt voulu éclairer un aspect du geste de théorisation de Benveniste, à partir de sa dimension terminologique. Reste qu'on peut se demander si cet aspect est conjoncturel ou bien associé en profondeur avec la théorie de l'énonciation.

\section{Prolongements pour conclure}

Pour tirer toutes les conséquences de cet exercice, il faudrait à présent l'appliquer à cette autre figure fondatrice de la linguistique de l'énonciation, qui en quelque sorte prend le relais chronologique de Benveniste à partir des années 1970, pour montrer que cet imaginaire politique n'est précisément pas le fait singulier de l'auteur du Vocabulaire, mais serait comme associé à la rhétorique théorique sur l'énonciation. L'hypothèse à défendre voudrait que, indépendamment des engagements politiques effectifs des linguistes, le geste même de réfléchir sur l'énonciation en France au tournant des années 1960-1970 convoquait nécessairement cet imaginaire politique.

Nous ne pourrons nous livrer à une telle enquête dans le cadre de cet article et nous bornerons à en signaler quelques pistes.

Que l'on songe par exemple à cette topique de la réalité du travail dissimulée derrière le quotidien le plus banal, lorsque Culioli termine son fameux article sur les "Valeurs modales et opérations énonciatives " par ce souhait: "Ainsi peut-on espérer retrouver, à travers la forme apparemment banale des énoncés quotidiens, les opérations enfouies du travail énonciatif. "(Culioli, 1990 : 155) Que l'on lise dans les propos plus vulgarisateurs de sa contribution à l'Encyclopédie des sciences de l'homme, sous le titre "La communication verbale " (Culioli, 1965), toute la section qu'il consacre au "rôle institutionnel du langage " et où l'on voit affleurer le plus clairement l'intertexte politique: le langage est dit "assure[r] la cohésion du groupe ", de même qu'il en reflète les diversifications « (selon les classes sociales, les activités techniques, l'origine géographique) " et, au final, "permet l'intégration, ou du moins l'insertion, du sujet dans le groupe, "socialise" l'être humain, et, par son caractère transindividuel, régularise nos conduites et notre adaptation à autrui. » (Culioli, 1965 : p. 71) Tout cela pourrait à la rigueur être lu aujourd'hui comme une série de généralités bien connues sur le langage, mais n'oublions pas que nous ne sommes alors qu'en 1965 et, surtout, précisons que cet extrait est suivi d'un passage où apparaissent deux termes forts de l'intertexte politique qui nous intéresse ici, " produit " et « appropriation » : 
Une langue est un véritable objet, un produit au sens fort du terme, qui accumule et généralise l'expérience socio-historique d'un groupe d'humains. Comme toute institution, le langage suppose un comportement individuel à l'intérieur d'un cadre, une attitude de rejet ou d'appartenance, une pratique par laquelle le sujet parlant s'approprie l'objet institutionnel, en l'adaptant à ses besoins. (ibid.)

On retrouve bien ici la même constellation terminologique, les mêmes harmoniques non pas forcément conceptuels, mais strictement rhétoriques, qui témoignent d'un ajustement du verbe du linguiste aux grandes topiques du discours social, en l'occurrence des topiques politiques.

Ces topiques invitent-elles à relire au même prisme les autres pans de la théorie linguistique d'un Benveniste ou d'un Culioli? Sans doute; c'est là la piste d'un autre travail.

\section{Bibliographie}

Althusser L. (1970), « Idéologie et appareils idéologiques d'État (notes pour une recherche) ", La Pensée, 151, repris dans Positions (1964-1975), 1976, Paris, Éditions sociales.

Barthes R. (1974) [1984], " Pourquoi j’aime Benveniste II », dans Le Bruissement de la langue: Essais critiques IV, Paris, Seuil (p. 194-196).

Benveniste É. (1966), Problèmes de linguistique générale, t. I, Paris, Gallimard, «Tel ».

- (1969), Vocabulaire des institutions indo-européennes, 2 t., Paris, Minuit.

- (1974), Problèmes de linguistique générale, t. II, Paris, Gallimard, "Tel ».

- (2012), Dernières leçons. Collège de France. 1968 et 1969, édition établie par Jean-Claude Coquet et Irène Fenoglio, préface de Julia Kristeva, postface de Tzvetan Todorov, Paris, Seuil/Gallimard, 2012.

Bronckart J.-P. \& Bota C. (2011), Bakhtine démasqué. Histoire d'un menteur, d'une escroquerie et d'un délire collectif, Genève, Droz.

Culioli A. (1965), " La communication verbale », dans L'homme et les autres, t. 4, Encyclopédie des sciences de l'homme: l'aventure humaine, Paris, Grange Batelière.

- (1990), Pour une linguistique de l'énonciation. Opérations et représentations, t. I, Paris, Ophrys.

Dessons G. (2006), Émile Benveniste, l'invention du discours, Paris, Éditions In Press. 
Dosse Fr. (1992), Histoire du structuralisme, 2 t. Paris, La Découverte.

Fenoglio I. (2011), « Déplier l'écriture pensante pour re-lire l'article publié. Les manuscrits de "L'appareil formel de l'énonciation" d'Émile Benveniste ", dans É. Brunet \& R. Mahrer (éds), Relire Benveniste. Réceptions actuelles des Problèmes de linguistique générale, Paris, L'HarmattanAcademia (p. 263-304).

Fodor F. (1996), «Antoine Meillet et les langues d'Europe: une manifestation de l'imaginaire linguistique des linguistes ", Travaux de linguistique, 7 , p. 131-140.

Houdebine A.-M. (1979), La variété et la dynamique d'un français régional (Poitou). Etudes phonologiques. Analyse des facteurs de variation à partir d'enquêtes à grande échelle dans le département de la Vienne (Poitou), Thèse de doctorat d'état, Paris-V, 3 vol.

Houdebine-Gravaud A.-M. (2002), «L'imaginaire linguistique: un niveau d'analyse et un point de vue théorique", dans A.-M. HoudebineGravaud (dir.), L'Imaginaire linguistique, Paris, L'Harmattan (p. 9-18).

Houdebine-Gravaud A.-M. (dir.) (2002), L'Imaginaire linguistique, Paris, L'Harmattan.

Jenny L. (2008), Je suis la révolution, Paris, Belin.

Laplantine C. (2011), "La poétique d'Émile Benveniste », dans É. Brunet \& R. Mahrer (éds), Relire Benveniste. Réceptions actuelles des Problèmes de linguistique générale, Paris, L'Harmattan-Academia (p. 71-95).

LTTR13 à paraître, "Benveniste serait-il aujourd'hui un linguiste de l'énonciation?", Actes du colloque Théories de l'énonciation. Benveniste après un demi-siècle.

- à paraître, "La subjectivité: lectures critiques entre grammaire et texte ", Estudos linguísticos / Linguistic Studies Journal.

Matonti F. (2011), «Entre Moscou et Prague: les premières réceptions des formalistes russes par les intellectuels communistes français (19671971) ", Langages, 182 (Théories du langage et politique des linguistes, par Jean-Louis Chiss), p. 69-81.

Michon P. (2010), Fragments d'inconnu. Pour une histoire du sujet, Paris: Éditions du Cerf, " Passages ».

Milner J.-Cl. (2008) [2002], Le Périple structural. Paris, Verdier. 
Ono A. (2007), La Notion d'énonciation chez Émile Benveniste, Limoges, Lambert Lucas.

Pavel Th. (1988), Le Mirage linguistique, Paris, Minuit.

Kristeva J. (2012), « Préface. Émile Benveniste, un linguiste qui ne dit ni ne cache, mais signifie ", dans Benveniste É. (2012), Dernières leçons. Collège de France. 1968 et 1969, édition établie par Jean-Claude Coquet et Irène Fenoglio, préface de Julia Kristeva, postface de Tzvetan Todorov, Paris, Seuil/Gallimard (p. 13-40).

Sebag L. (1964), Structuralisme et marxisme, Paris, Payot.

Sériot P. (2011), "Vološinov, la philosophie du langage et le marxisme ", Langages, 182 (Théories du langage et politique des linguistes, par JeanLouis Chiss), p. 83-97.

Tournier M. (2007), Les mots de mai 1968, Toulouse, Presses universitaires du Mirail.

Vološinov V. (1930), "Konstrukcija vyskazyvanija », Literaturnaja ucheba, 3, p. 65-87.

- ([1929]2010), Marxisme et philosophie du langage, texte édité et traduit par Patrick Sériot et Inna Tylkowski-Ageeva, Limoges, Lambert Lucas.

Article reçu en août 2012. Pas de révision. 